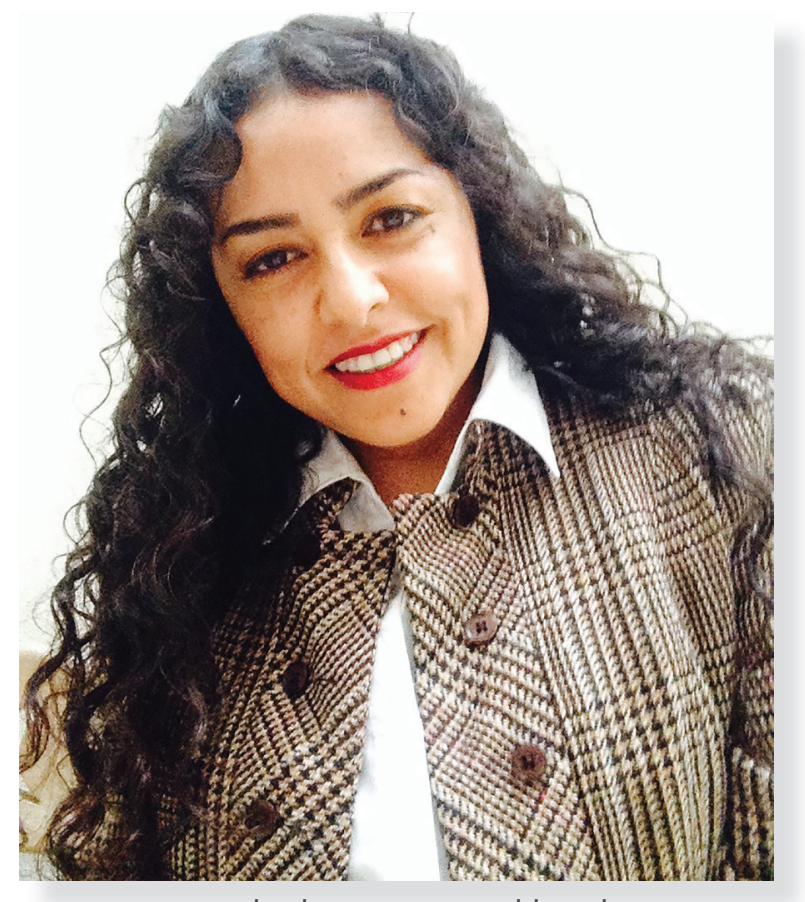

Katia Aleyda Manrique Maldonado*

\section{La comunicación organizacional: elemento fundamental para el éxito de una institución de Educación Superior}

\author{
Organizational communication: fundamental \\ element for the success of a higher Education \\ Institution
}

Recibido: 01-06-15 Aprobado: 28-10-15

\title{
Resumen
}

La comunicación organizacional es un elemento importante para el impulso, crecimiento y posicionamiento de todas las organizaciones, realidad de la que no escapan las instituciones educativas. Sin embargo son las que quizá apliquen menos estrategias y programas de gestión de comunicación que permitan impulsarlas y posicionarlas a diferencia de las miles de empresas con fines lucrativos. El siguiente artículo está basado en una investigación que tiene por objetivo analizar la importancia de la gestión de comunicación al interior de una institución de educación superior y cómo influyen estos procesos en el logro de los objetivos organizacionales, para lo cual se utilizaron fuentes teóricas y trabajo de campo. El artículo finaliza con directrices de gestión de comunicación para una institución educativa perteneciente a una universidad pública.

\section{Abstract}

Organizational Communication is an important element for the impulse, growth and positioning of all organizations, a reality public institutions cannot avoid; nevertheless, they are the ones that perhaps apply less strategies and programs in communication management. The following article is based on a research study which main objective was to analyze the importance of communication management within a higher education institution and how these processes influence the achievement of organizational objectives. Theoretical sources and field work were used. The article ends with communication management guidelines for a public higher education institution.

Palabras clave

comunicación y educación; comunicación organizacional; gestión de comunicación en la educación

\section{Keywords}

communication and education, organizational communication, communication management

*Katia Aleyda Manrique Maldonado: Licenciada en Ciencias de la Comunicación, por la Universidad Autónoma de Guerrero, México. Master en Ciencias de la Comunicación, mención: Comunicación Organizacional por la Universidad de La Habana, Cuba. En proceso de titulación del doctorado en Comunicación Social por la misma universidad. Profesora de la Universidad Autónoma de Guerrero desde 1999. Para contactar a la autora: katiamm1@yahoo.com.mx 
Las universidades públicas en México se han visto en la necesidad de implementar programas de gestión de comunicación que permitan contribuir a su crecimiento y fortalecimiento, primeramente porque refuerzan los flujos de comunicación al interior de ellas y ayudan a cumplir con los objetivos institucionales, y en segunda porque de manera externa contribuyen entre otras cosas a la conformación de una imagen pública positiva. De esta manera posicionan sus diferentes ofertas educativas y a sus egresados en un terreno ampliamente competitivo.

Los objetivos del presente artículo son visualizar los conceptos básicos de la comunicación desde la perspectiva organizacional, analizar la importancia de la gestión de la comunicación dentro de las instituciones educativas consideradas como organizaciones sociales, así mismo proponer directrices enfocadas a reforzar estos procesos de gestión.

Se tomó como unidad de análisis a la Unidad Académica de Comunicación y Mercadotecnia de la Universidad Autónoma de Guerrero, México, como parte de una investigación de tipo mixta, ya que es resultado de un análisis teórico y de un estudio cuantitativo y cualitativo.

\section{Conceptualizaciones teóricas}

\section{Comunicación y organización}

Son muchos los teóricos que a partir de los estudios de la comunicación organizacional han elaborado definiciones sobre el concepto de organización: desde Max Weber a principios del siglo pasado y sus aportes al tratar de plantear una forma de organización que fuera más eficiente y eficaz a las necesidades de la sociedad, hasta teóricos contemporáneos.

Goldhaber (1991) apunta que una organización es definida como una red de relaciones interdependientes y debido a la naturaleza de sus interacciones con el medio ambiente es un sistema social abierto. Para Schein (1998, p. 24) "las empresas son en sí mismas sistemas abiertos en constante interacción con sus distintos medios y se componen además de muchos subgrupos, unidades laborales, grados jerárquicos y áreas geográficas dispersas" de acuerdo a estas definiciones se puede decir que las organizaciones son sistemas sociales que tienen objetivos y metas específicas, están enconstante movimiento y vinculadas necesariamente con el ambiente.
Las instituciones de Educación Superior son organizaciones con características definidas cuyos objetivos fundamentales son formar profesionales en diferentes áreas del saber, desarrollar la investigación y fomentar la vinculación. Estas instituciones tienen la función social de generar, transmitir y difundir el conocimiento.

Después de definir a las organizaciones y ubicar el papel de las instituciones educativas ¿Cuál debe ser la importancia de la comunicación y el rol que juega dentro de ellas? La comunicación es un proceso vital, presente en cualquier relación social, se puede definir como un intercambio de información y significados entre los seres humanos, donde la retroalimentación es fundamental para una mejor comprensión de los mismos. Para Narváez y Campillo (2011, p. 63) "la comunicación se entiende como el proceso básico de interacción en el funcionamiento de las organizaciones, a través del cual se hace posible compartir mensajes, significados y conductas en un marco social específico". Elemento necesario e imprescindible en cualquier sistema social.

De acuerdo con Katz y Kahn (1999, p. 250) "La comunicación es un proceso social de suma importancia para el funcionamiento de cualquier grupo, organización o sociedad; es posible resumir en ellas formas de interacción grupal como son influencia, cooperación, contagio o imitación social y liderazgo". De acuerdo a esta concepción no se pueden visualizar a las instituciones educativas sin este proceso vital para el desarrollo de cada una de sus acciones, desde la retroalimentación de información en las aulas entre maestros y alumnos, dentro de las funciones del aparato administrativo, hasta en el contacto de los centros educativos con su contexto, esto último es frecuente gracias a la vinculación que deben de tener con los sectores sociales.

\section{Comunicación interna}

Las organizaciones no podrían existir sin el intercambio diario de información al interior de ésta; los flujos de comunicación, los medios utilizados, el contenido de los mensajes son vitales para el cumplimiento de los objetivos organizacionales. Sin embargo, no en todas las universidades están conscientes de la importancia que tiene la comunicación interna para el crecimiento y desarrollo de una organización ni destinan presupuesto para reforzarla y mantenerla como algo vital.

Para Lucas (1997) los procesos comunicativos en el interior del sistema organizativo constituyen la 
comunicación interna, dirigida a conseguir una estabilidad en la organización con vistas a que se alcancen sus fines. Mientras tanto Andrade (2012, p. 12) señala que "la comunicación interna es el conjunto de actividades efectuadas, por cualquier organización para la creación y mantenimiento de buenas relaciones con y entre sus miembros a través del uso de diferentes medios de comunicación que los mantengan informados, integrados y motivados para contribuir con su trabajo al logro de objetivos organizacionales". Es importante aclarar que al interior de una institución educativa todos los que forman parte de su organigrama integran el público interno. Sus miembros están estrechamente interconectados y puede existir una relación de dependencia laboral directa. Este público está conformando por directivos, docentes, administrativos, alumnos y personal de intendencia.

Los flujos de comunicación que circulan entre el público interno son vitales para el buen funcionamiento de una institución, ya que es a través de ellos que se envía información de tareas a realizar, se difunden reglamentos, se refuerza la cultura interna, se elaboran nuevos planes y programas para la organización, se intercambian puntos de vista entre trabajadores y se fortalece también la comunicación informal entre todos los integrantes.

\section{Comunicación externa}

La comunicación externa es la información que genera la institución educativa y se envía a sus diferentes públicos externos, como pueden ser, escuelas de la competencia, medios de comunicación, instituciones gubernamentales, empresas, asociaciones, centros de investigación, ciudadanía en general, entre otros. La comunicación interna y externa están estrechamente vinculadas pues todo lo que la organización emita al exterior forzosamente será información generada y procesada al interior de la misma por su público interno. De esta manera son dos elementos que no deben visualizarse por separado sino interrelacionados y gestionados de forma conjunta de manera permanente.

\section{Aspectos referenciales y metodológicos}

La Unidad Académica de Comunicación y Mercadotecnia (UACOM) el elemento de análisis para esta investigación es una institución educativa de nivel superior, perteneciente a la Universidad Autónoma de Guerrero (UAGro), México. Está ubicada en la ciudad de Chilpancingo y tiene actualmente 788 estudiantes en las dos licenciaturas con las que cuenta, las cuales son: la Licenciatura en Ciencias de la Comunicación y la Licenciatura en Mercadotecnia.
En este momento a nivel estatal existen cuatro universidades privadas que ofertan los mismos estudios.

Desde el punto de vista metodológico la investigación está sustentada en una indagación de tipo mixta ya que se utilizó el diseño cuantitativo y cualitativo, para poder obtener contrastes y puntos de vistas con instrumentos distintos que permitan ahondar más en el tema. Por lo tanto, se aplicó una encuesta a maestros y alumnos de la institución educativa y se manejaron entrevistas estructuradas al director de la UACOM, así como a estudiantes y egresados de la misma Unidad Académica, lo que permitió describir, analizar y profundizar en el objeto de estudio. Se tomó una muestra aleatoria para la encuesta con un margen de confianza del 95.5 por 100 lo que nos permitió trabajar con información estadísticamente confiable.

\section{Resultados}

En toda organización el público interno es determinante y esencial para su funcionamiento y desarrollo, para el presente estudio se aplicó una encuesta dentro del público interno más numeroso de la UACOM como son los estudiantes. Lo anterior para tener un diagnóstico de sus procesos de comunicación y conocer su percepción en cuanto a imagen y comunicación de la misma unidad, igualmente se aplicó una encuesta a docentes de base.

Lograr el bienestar del público interno debe ser uno de los objetivos primordiales de toda organización, así como conocer las necesidades, deseos, complicaciones e insatisfacciones de manera oportuna, proporciona herramientas para encontrar sus soluciones y evitar problemáticas mayores.

Es por eso que algunos de los indicadores de las encuestas y entrevistas están enfocados a detectar el nivel de satisfacción de los estudiantes de la UACOM, detectándose que el $56.9 \%$ de ellos se sienten satisfechos de pertenecer a la institución, sin embargo el $25.5 \%$ es un porcentaje alto para considerar que en éste indicador están los alumnos que consideran que les es indiferente y no están satisfechos de estudiar en ella.

Una gran cantidad de problemas en las instituciones educativas son resultado de procesos de comunicación deficientes en toda su estructura interna, lo que puede influir en el clima laboral, la identidad, y su cultura.

Al indagar sobre el papel de la comunicación entre la dirección de la escuela y los estudiantes, el $30.59 \%$ consideran que la comunicación es muy buena, sin embargo contrasta con el $69.4 \%$ donde se encuentran 
los alumnos que visualizan a la comunicación como escasa, deficiente e incluso los que consideran que no existe comunicación. Esto último cuando consideran que no hay contacto alguno con la dirección. (Ver gráfico 1)

Con respecto a los docentes, los porcentajes no están tan alejados: el 38. $1 \%$ manifiesta que la comunicación en muy buena, mientras que el $61.9 \%$ de los profesores manifiesta que la comunicación es escasa y deficiente.

Los porcentajes proyectados en estudiantes y docentes son similares, no obstante, en entrevista el director de la escuela considera que la comunicación con los profesores es permanente, enfatizando reuniones periódicas para distribuir la carga laboral o de tipo académico, donde los docentes realizan la planeación del trabajo en las aulas.

En relación a la comunicación entre maestros y alumnos, los docentes tienen una percepción positiva ya que el $76.19 \%$ manifestaron que es buena, al respecto el $65.88 \%$ de los estudiantes encuestados opinaron lo mismo de la comunicación con sus docentes, sin embargo el $24.71 \%$ de este último grupo la percibe como deficiente (ver gráficos 2 y 3 ).

Estos dos grupos tienen cierta coincidencia con respecto a la comunicación que existe entre ellos, pero podría elevarse ese indicador ya que aunque el porcentaje es menor hay quienes la consideran deficiente y mala. El reto es lograr reducir al máximo ese tipo de percepciones para alcanzar un nivel de satisfacción alto.

\section{Gráfico 1}

La comunicación entre la dirección de la escuela y los estudiantes (percepción estudiantil)

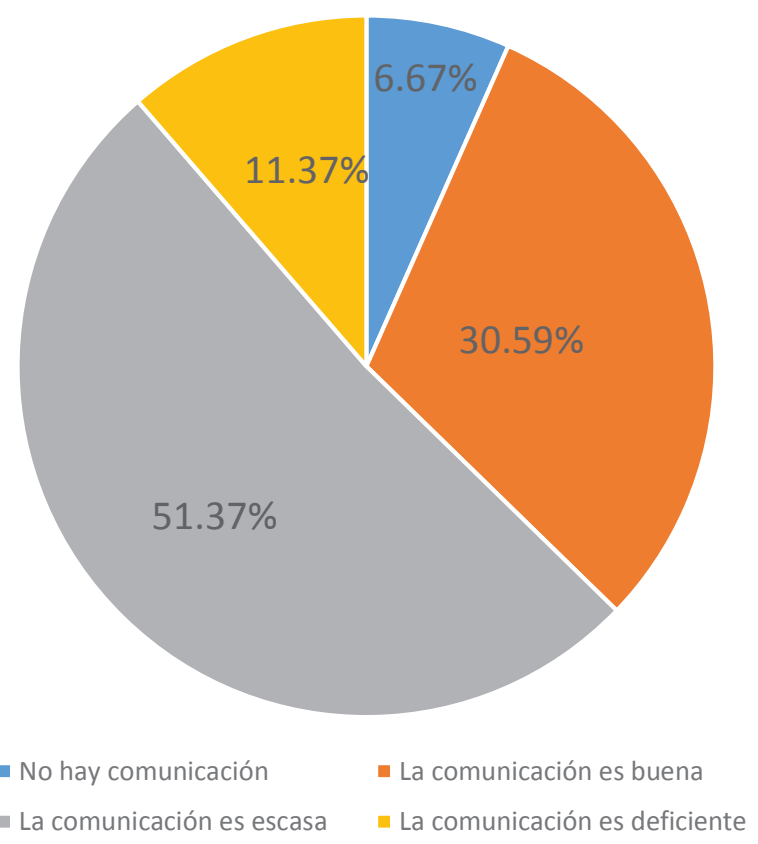

\section{Gráfico 2}

\section{La comunicación entre maestros y alumnos (percepción docentes)}

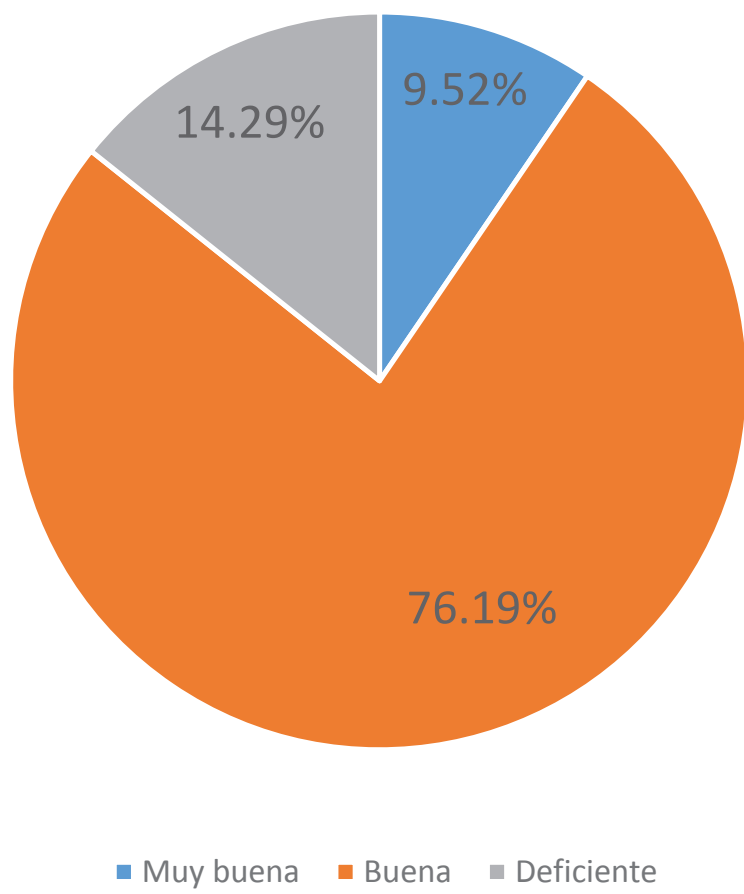




\section{Gráfico 3}

\section{La comunicación entre maestros y alumnos} (percepción alumnos)

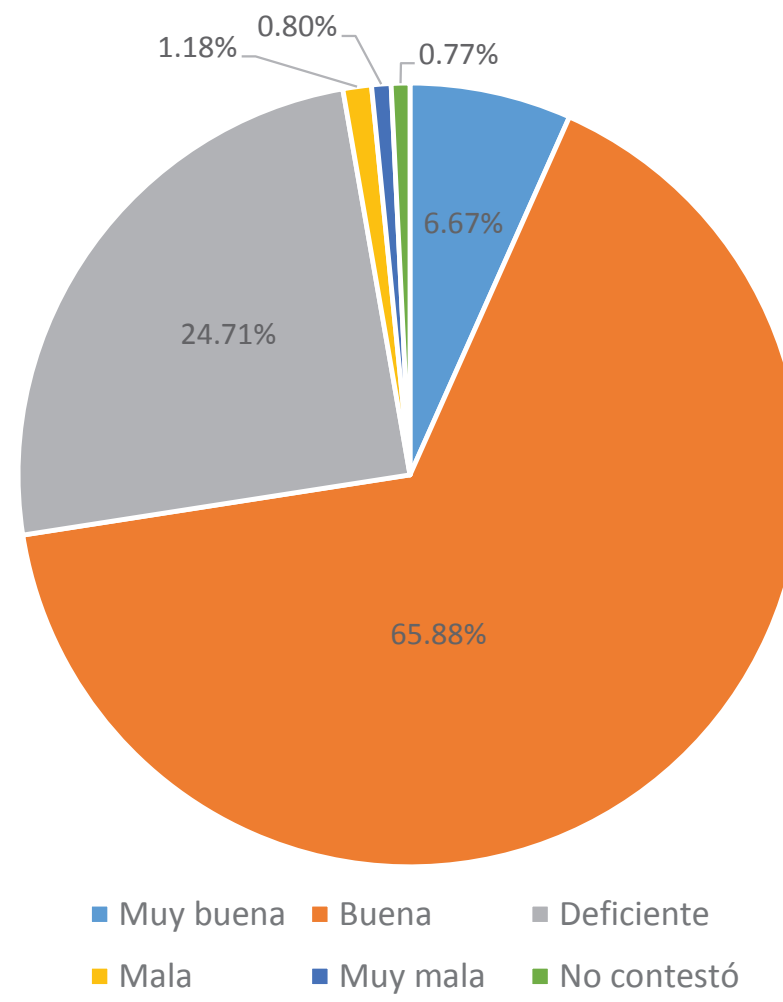

Dentro de la Unidad Académica, no existen grupos fijos de estudiantes ya que ellos tienen la facilidad de seleccionar sus unidades de aprendizaje y horarios, esto motiva que tengan que cambiar constantemente de salón y por consiguiente de compañeros de clase, por lo cual los alumnos en entrevistas manifiestan tener poca oportunidad de estrechar y consolidar lazos de amistad.

De la misma manera los alumnos entrevistados manifiestan que los flujos de comunicación al interior de la Unidad Académica se ven alterados por cuestiones políticas, sobre todos si se vive un proceso electoral para la elección de director o rector, la interacción después de un evento de este tipo se vuelve más lenta, y la comunicación formal e informal es deficiente.

Se detectó que no existe en el organigrama un departamento que esté dedicado a la comunicación organizacional o las relaciones públicas, ni tampoco estas actividades están incluidas como funciones secundarias en algún otro. En el mismo sentido la Institución no cuenta con un Manual de Gestión de Comunicación, y las acciones que se requieren se van desarrollando de acuerdo a lo que necesita la institución en su momento. Por consiguiente se visualiza que las actividades en comunicación que se realizan no están vinculadas a un programa de gestión, ni tampoco a objetivos generales, sino que se identifican como inconexas.

Al revisar y analizar la página Web de la escuela como uno de los medios de comunicación internos podemos advertir que está vinculada al sitio web de la Universidad Autónoma de Guerrero. La información destinada a los estudiantes se refiere a la selección de unidades de aprendizajes, horarios y calendario de exámenes para su público interno, así como información general de las dos licenciaturas que se ofertan. A pesar de que se encuentra la filosofía de la Unidad los estudiantes no se la saben, incluso en las entrevistas estructuradas los alumnos manifiestan que la página no está actualizada del todo y no tiene la información requerida. Los egresados entrevistados manifiestan que la consultaban poco como estudiantes y que no abren la página web desde que salieron de la institución.

Es importante mencionar que no se detectó la posibilidad de contacto o retroalimentación con el visitante mediante algún medio, como formularios o chat en línea de manera interactiva, no obstante en la parte inferior central de página web analizada, se puede observar tanto la dirección, teléfono y correo electrónico en tipografía pequeña.

La Unidad Académica tiene una cuenta de Facebook institucional, utilizada para reforzar los flujos de comunicación interna con mensajes permanentes para docentes y estudiantes. Con respecto a este medio los estudiantes expresan que se encuentran satisfechos con su función ya que hay publicaciones constantes por parte de la institución, y es el medio de comunicación más utilizado para solicitar información. Sin embargo manifiestan una clara necesidad de comunicación personal o cara a cara con los directivos.

En general los alumnos distinguen una imagen positiva de la escuela, aunque pudiera mejorarse ya que un $44.3 \%$ visualizan a su institución educativa como aceptable, de igual manera consideran que la formación educativa de los estudiantes en la UACOM es buena, e incluso el $87.1 \%$ de los estudiantes conciben que los maestros están en un buen parámetro desde el punto de vista académico. En las entrevistas los egresados manifiestan tener una percepción positiva de la UACOM al considerarla una buena institución educativa. No obstante puede reforzarse la percepción y mejorarla con estrategias enfocadas entre otras cosas a difundir sus logros y avances y al integrar al público interno en todos sus procesos. 
Sin embargo, la gestión de comunicación interna no se percibe dentro de la UACOM como una actividad primordial, pues no se detecta un departamento que se encargue de administrar estas funciones, diagnósticos que permitan evaluar la realidad de la institución en materia de comunicación, identidad o imagen, ni planes a seguir en este sentido. Prevalece por lo tanto una comunicación lineal e inconexa, que puede influir en la motivación, identidad y satisfacción que el público interno pueda tener.

\section{Conclusiones}

Dentro de los principales retos de las universidades se encuentra cumplir sus objetivos y acciones planteadas en su misión, así como la visión proyectada, para lograrlo requieren de un trabajo permanente, de la implementación de programas, acciones y estrategias de comunicación encaminadas de manera conjunta para el logro de fines. Hoy en día no basta con tener los mejores planes y programas de estudio, hay que saber difundirlos para de este modo posicionarse, manteniendo una imagen y una reputación positiva, así como un público interno satisfecho.

No hay que olvidar que la comunicación externa está estrechamente vinculada con los parámetros de comunicación interna que se manejan en cualquier institución. Las estrategias y acciones de comunicación interna no son aspectos aislados ni debe por ningún motivo trabajarse por separado, ya que tiene que existir congruencia entre la comunicación que fluye al interior con lo que la organización emite al exterior. Lo anterior incide en la credibilidad y confianza de ambos públicos.

La difusión permanente de todas las actividades que merecen propagarse, así como los logros de la institución, estimulan la credibilidad y confianza en los públicos de la organización e impactan en la reputación e imagen pública.

Por lo anterior se proponen las siguientes directrices para reforzar la comunicación interna de la institución educativa.

- Iniciar un proceso de capacitación y formación en el área de la comunicación organizacional, que permita que el aparato administrativo de la Unidad Académica visualice el valor de la gestión de comunicación para el crecimiento y desarrollo de la misma y lo implemente como un factor imprescindible en todos sus procesos.

- Realizar el respectivo Plan de Comunicación que permitirá entre otras cosas reforzar la identidad, la cultura y la imagen interna. Estos aspectos fortalecerán su pertenencía y arraigo del público interno y lo harán fuerte frente a futuras crisis. Se deben incluir estrategias enfocadas a mejorar o fortalecer el clima laboral de los maestros, este a su vez repercutiría en el ambiente educativo dentro del proceso de enseñanza aprendizaje en las aulas.

No hay que olvidar que todas las acciones en comunicación, tienen que ir enfocadas a contribuir con los objetivos organizacionales que posee la institución educativa.

- Es de suma importancia aplicar los diagnósticos en comunicación de manera periódica, aplicando técnicas e instrumentos que evalúen el estado de la comunicación al interior de la institución educativa, para poder detectar entre otras cosas, flujos y barreras de comunicación, efectividad de los medios internos de comunicación utilizados y evaluar la imagen, cultura e identidad. Igualmente se deben utilizar instrumentos para medir el impacto de las estrategias que han sido aplicadas, de no ser positivos los resultados planear los cambios respectivos.

La gestión de comunicación interna es un recurso intangible en una institución educativa que permite el avance y logro de las metas establecidas, del mismo modo contribuye en la motivación, satisfacción e integración de los grupos de interés internos, reforzando el sentido de pertenencia, así como su cultura, imagen e identidad.

\section{Referencias}

Andrade H. (2012) Definición y alcance de la comunicación organizacional. En C. Collado (coordinador), La comunicación en las organizaciones. (pp. 11,17) México: Trillas.

Goldhaber G. (1991). Comunicación Organizacional. México: Diana.

Katz D. \& Kahn R. (1999). Psicología Social de las Organizaciones. México: Trillas.

Lucas M. A. (1997). La comunicación en las empresas y en las organizaciones. España: Bosch comunicación.

Narváez L. \& Campillo I. (2011) Transformación de las organizaciones. El papel estratégico de la comunicación. En A. Rebeil (coordinadora), La comunicación estratégica en las organizaciones. México: Trillas

Schein E. (1998). La Cultura Empresarial y el Liderazgo. Una visión dinámica. España: Plaza y Janés. 